Check for updates

Cite this: RSC Adv., 2017, 7, 46236

Received 24th July 2017

Accepted 19th September 2017

DOI: 10.1039/c7ra08173a

rsc.li/rsc-advances

\section{Flame-retardant effect of a phenethyl-bridged DOPO derivative and layered double hydroxides for epoxy resin $\dagger$}

\author{
Wei Yan, (D) *abc Jie Yu, ${ }^{\star b}$ Mingqiu Zhang, ${ }^{c}$ Shuhao Qin, ${ }^{b}$ Tao Wang, ${ }^{a}$ Weijiang Huang ${ }^{b}$ \\ and Lijuan Long ${ }^{b}$
}

Flame-retardant epoxy resin (EP) composites were prepared by the incorporation of a phenethyl-bridged DOPO derivative (DiDOPO) and modified layered double hydroxide (OLDH). In addition, the flameretardant behaviour, thermal stability, synergism between DiDOPO and OLDH, and mechanical properties of the flame-retardant EP composites were examined. The introduction of a specific amount of OLDH in the intumescent flame-retardant EP led to considerable enhancement in flame retardancy, thermal stability, and mechanical properties. Furthermore, the effects of DiDOPO and OLDH on the flame-retardant properties of the EP composites were characterised by the limiting oxygen index (LOI) as well as the UL-94 vertical burning and cone calorimeter tests (CCT). The LOI of the EP/DiDOPO5/OLDH5 composites increased from $21.8 \%$ to $31.5 \%$, and the composites exhibited the $\mathrm{V}-0$ rating in the UL-94 vertical burning test. Moreover, the EP/DiDOPO5/OLDH5 composite exhibited the highest tensile strength and a low peak heat release rate and the total heat release values. In addition, scanning electron microscopy observation revealed a considerably more continuous, compact char residue for the EP/DiDOPO/OLDH composite. Thermogravimetric analysis and CCT revealed that DiDOPO and OLDHs exert gas- and condensed-phase flame-retardant effects. Overall, different flame retardant performance is related to the characteristics of each composite; dispersion state in the EP matrix; and structural changes during burning.

\section{Introduction}

Epoxy resins (EP) have been widely applied as adhesive agents, coatings, and laminated composites because of their desirable properties, including low shrinkage during curing, excellent mechanical strength, superior insulation and dielectric properties, and satisfactory resistance to moisture and organic solvents. ${ }^{1-3}$ Unfortunately, because of their flammability, their use in some fields (such as electronic industries) that require high flame resistance is limited. ${ }^{4-7}$ Meanwhile, some serious issues, such as the flammability of EP, restrict its application in the aforementioned field. Thus, it is crucial to develop a flame retardant for EP systems. Conventionally, halogen compounds are used for improving the flammability of EP. The incorporation of certain flame-retardant additives, including phosphoro $^{\mathbf{8 - 1 0}}$ and silicon-containing compounds, ${ }^{\mathbf{1 1}, 12}$ functionalised

${ }^{a}$ School of Chemistry and Materials Engineering, Guiyang University, Guiyang, 550005, PR China. E-mail: lrasyw@163.com

${ }^{b}$ National Engineering Research Center for Compounding and Modification of Polymer Materials, Guiyang 550014, PR China. E-mail: yujiegz@126.com 'School of Chemistry, Sun Yat-sen University, Guangzhou 510275, PR China

$\dagger$ Electronic supplementary information (ESI) available. See DOI: $10.1039 / \mathrm{c} 7 \mathrm{ra} 08173 \mathrm{a}$ layered double hydroxide (LDH) fillers, ${ }^{\mathbf{1 3 - 1 5}}$ and graphene-based fillers, ${ }^{7,16,17}$ can effectively improve the flame retardancy of EP.

Derivatives based on 9,10-dihydro-9-oxa-10-phosphaphenanthrene-10-oxide (DOPO) as a type of phosphorus-containing flame retardant is a promising alternative for a flame retardant. Several studies have reported that the high activity of DOPO derivatives is related to the production of $\mathrm{PO}^{\circ}$ radicals by a gaseous-phase mechanism..$^{\mathbf{1 0 1 8 - 2 0}}$ Angell et al. ${ }^{21}$ have synthesised an ethylene-bridged DOPO derivative, referred to as $\mathrm{DOPO}_{2}$-ethylene. The formation of this derivative blocks the reactivity and increases the thermal stability of DOPO-based compounds. The UL-94 test results revealed that the incorporation of $\mathrm{DOPO}_{2}$-ethylene into EP leads to good flame-retardant properties. Qian et al. ${ }^{22}$ have synthesised a novel DOPO derivative containing two bridged chemical bonds between phosphaphenanthrene and triazine-trione groups. The loading of only $4 \mathrm{wt} \%$ of the newly synthesised DOPO derivative gave a UL94 V-0 rating for the EP thermoset with a limited oxygen index (LOI) of $35.9 \%$. Previously, our group has carried out studies on a phenethyl-bridged DOPO derivative incorporated into polylactic acid. With a loading of $15 \mathrm{wt} \% \mathrm{DOPO}_{2}$-phenethyl, an LOI of $28.2 \%$ and a UL-94 V-0 rating are obtained. ${ }^{23}$ Thus, it is crucial to design reasonably functional DOPO-based flame retardants for several applications. 
LDHs have attracted increasing attention as a new generation of environment-friendly, highly efficient flame retardants for polymers because of their tuneable chemical composition and layered structures. ${ }^{24}$ Magnesium hydroxide and alumina hydroxide were used as inorganic fillers, which initiate an endothermic reaction during combustion, leading to the decreased surface temperature with the release of free water molecules. The concentration of combustible volatiles decreases because of a protective layer formed by the filler composites. In addition, magnesium aluminium layered double hydroxide (MgAl LDH) was more effective than $\mathrm{Mg}(\mathrm{OH})_{2}$ and $\mathrm{Al}(\mathrm{OH})_{3}$ as a thermal stabiliser and flame retardant, respectively. High loading of all inorganic compounds was crucial to achieve flame retardant standards. LDH nanofillers served as a thermal barricade by acting as an endothermic heat sink in the condensed phase during combustion. To achieve high flame retardancy levels, the combination of different flame retardants in minimum doses was used to satisfy the required industrial standards. The synergistic effects of different inorganic fillers, including clay and organophosphate nanocomposites, were examined. The synergistic effects of organophosphate and modified LDH in an EP composite were examined, and modified LDH exfoliated when it was blended with EP and organophosphate, and the char residue of the composite was characterised: A $\mathrm{P}-\mathrm{O}-\mathrm{H}$ absorption band was observed, indicating the formation of a phosphoric acid derivative during char formation. ${ }^{25}$

The thermal and flammability properties of EP were examined using graphene/NiFe $\mathrm{LDH}$ and a multi-intercalating system in $\mathrm{LDH}^{\mathbf{1 3 , 2 6 , 2 7}}$ In the presence of melamine polyphosphate and boric acid, phenyl-phosphonate-intercalated magnesium aluminium LDH exhibited a synergistic effect for improving the thermal and flame retardancy of the ethylenevinyl acetate (EVA) composite. ${ }^{28}$ Synergistic effects of LDH in the presence of a phosphorus-nitrogen intumescent flame retardant and ammonium polyphosphate in PP- and EPDMblended composites have been reported..$^{29,30}$ For achieving a good intumescent effect, DiDOPO was used herein. Generally, magnesium and aluminium hydroxides were used as inorganic flame retardants because of their high heat sink capacities; therefore, MgAl LDH is selected as the additive. MgAl LDH with a molar ratio of $2: 1$ was selected to enhance the surface charge density, which increased the intercalation of sodium dodecylbenzene sulfonate (SDBS) surfactant molecules. MgAl LDH was intercalated with SDBS to increase the spacing between layers and increase the hydrophobic nature, which increased the compatibility of this composite with the organic polymer epoxy resin.

To reduce the LDH loading while simultaneously maintaining or improving the flame retardant efficiency, one method involves the combination of fillers, such as DOPO derivatives together with LDH. A phenethyl-bridged DOPO derivative (DiDOPO) and organomodified MgAl-LDHs (OLDH) were used to examine the flame retardance of EP. The thermal and flammability properties were systematically investigated with respect to the variation in the $\mathrm{LDH}$ and DiDOPO whose concentrations of the epoxy composites. Furthermore, different synergistic effects of DiDOPO and LDHs on the flame retardancy of EP were comprehensively investigated. The underlying flame-retardant mechanisms were analysed and discussed. This study aided in the optimization of compositions for the minimum dose of DOPO-derived flame retardants.

\section{Experimental}

\subsection{Materials}

Metal nitrate salts $\mathrm{Mg}\left(\mathrm{NO}_{3}\right)_{2}$ and $\mathrm{Al}\left(\mathrm{NO}_{3}\right)_{3}$ used for the synthesis of MgAl-LDH were obtained from Tianjin Kemi Ou Chemical Reagent Co., Ltd., China. SDBS was obtained from Sinopharm Chemical Reagent Co., Ltd., China. The diglycidyl ether of bisphenol-A (DGEBA, epoxide value of $0.48-0.52 \mathrm{~mol} / 100 \mathrm{~g}$, commercial name: E-51) was purchased from Laizhou Baichen Insulation Materials Co. Ltd., China. The curing agent 2-ethyl-4methylimidazole (EMI-2,4) was purchased from Tianjin Weiyi Chemical Technology Co., Ltd., China. The flame retardant phenethyl-bridged DOPO derivative (DiDOPO) was synthesised in our laboratory according to a previously reported method. ${ }^{31}$ Fig. S1† shows the chemical structure of DiDOPO. The other reagents were obtained from Chongqing Chuandong Chemical Group Co., Ltd.

\subsection{Organic modification of LDH}

Organically modified MgAl-LDHs (OLDH) were synthesised in one step as follows. First, $\left[\mathrm{Mg}\left(\mathrm{NO}_{3}\right)_{2} \cdot 6 \mathrm{H}_{2} \mathrm{O}\right.$ and $\left.\mathrm{Al}\left(\mathrm{NO}_{3}\right)_{3} \cdot 9 \mathrm{H}_{2} \mathrm{O}\right]$ with a $\mathrm{Mg}^{2+}: \mathrm{Al}^{3+}$ molar ratio of $2: 1$ were dissolved in water and at a total metal ion concentration of $0.3 \mathrm{M}$. Second, this solution was added to an aqueous solution of $0.12 \mathrm{M}$ SDBS. The mixed solution was heated under continuous stirring at a constant reaction temperature of $50{ }^{\circ} \mathrm{C}$. During the synthesis, the $\mathrm{pH}$ was maintained at 9-10 by the addition of a suitable amount of a $1.5 \mathrm{M} \mathrm{NaOH}$ solution. After the addition of the mixed metal salt solution, the resulting slurry was continuously stirred at the same temperature for $0.5 \mathrm{~h}$ and then allowed to age in a heater at $80{ }^{\circ} \mathrm{C}$ for $12 \mathrm{~h}$. The final products were filtered and washed several times with distilled water to wash out any unreacted surfactant molecules until the $\mathrm{pH}$ of the supernatant solution was approximately 7 . Then, the material was dried in the oven at $70{ }^{\circ} \mathrm{C}$ until constant weight. Hereafter, this material will be referred to as organomodified LDH (OLDH). ${ }^{32,33}$ Fig. S2 and $\mathrm{S} 3 \uparrow$ shows the X-ray diffraction (XRD) patterns and Fourier transform infrared (FTIR) spectra of $\mathrm{LDH}$ and $\mathrm{OLDH}$, respectively.

\subsection{Preparation of flame-retardant EP composite}

The DiDOPO powder was evenly dispersed into EP under mechanical stirring at room temperature. Next, the mixture was slowly heated to $130{ }^{\circ} \mathrm{C}$ and stirred until DiDOPO was completely dissolved in EP. For the EMI-2,4 curing system, the EP/DiDOPO mixture was cooled to $50{ }^{\circ} \mathrm{C}$, and EMI-2,4 was then added into the mixture and adequately blended to form a uniform hybrid. After the hybrid was degassed for $20 \mathrm{~min}$ in a vacuum oven at $40{ }^{\circ} \mathrm{C}$, it was poured into preheated moulds 


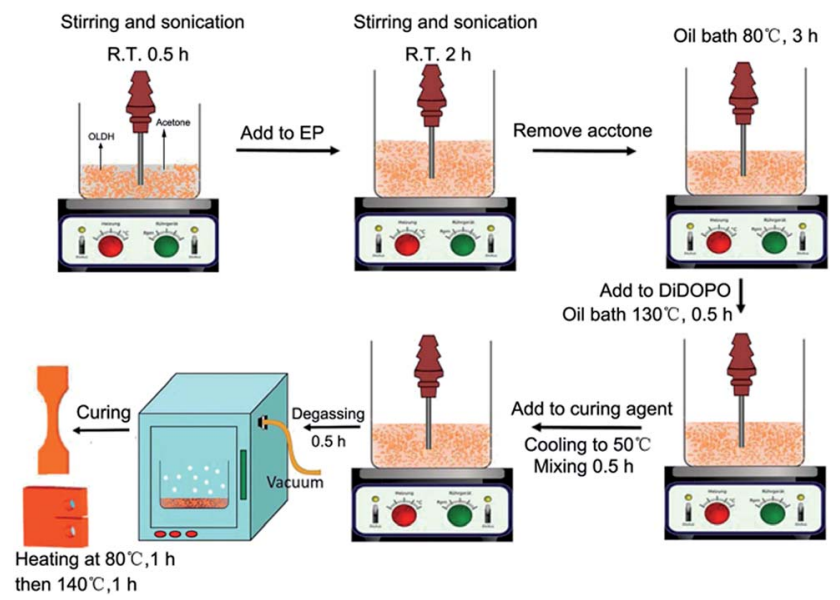

Fig. 1 Preparation of the EP/DiDOPO/OLDH composites.

Table 1 Compositions and flame retardant parameters of EP composites

\begin{tabular}{|c|c|c|c|c|c|}
\hline \multirow[b]{2}{*}{ Sample } & \multicolumn{3}{|c|}{ Composition (wt\%) } & \multicolumn{2}{|c|}{$\begin{array}{l}\text { Flame } \\
\text { retardance }\end{array}$} \\
\hline & $\mathrm{EP}$ & DiDOPO & $\mathrm{OLDH}$ & LOI $(\%)$ & UL-94 \\
\hline EP & 100 & 0 & 0 & 21.8 & N.R. \\
\hline EP/DiDOPO1 & 100 & 1 & 0 & 24.1 & $\mathrm{~V}-2$ \\
\hline EP/DiDOPO5 & 100 & 5 & 0 & 35.8 & $\mathrm{~V}-0$ \\
\hline EP/DiDOPO10 & 100 & 10 & 0 & 38.0 & V-0 \\
\hline EP/OLDH1 & 100 & 0 & 1 & 21.9 & N.R. \\
\hline EP/OLDH5 & 100 & 0 & 5 & 23.6 & V-0 \\
\hline EP/OLDH10 & 100 & 0 & 10 & 22.1 & V-0 \\
\hline EP/DiDOPO0.5/OLDH0.5 & 100 & 0.5 & 0.5 & 25.2 & $\mathrm{~V}-0$ \\
\hline EP/DiDOPO2.5/OLDH2.5 & 100 & 2.5 & 2.5 & 27.8 & $\mathrm{~V}-0$ \\
\hline EP/DiDOPO5/OLDH5 & 100 & 5 & 5 & 31.5 & V-0 \\
\hline
\end{tabular}

and cured at $80^{\circ} \mathrm{C}$ for $1 \mathrm{~h}$ and then at $140{ }^{\circ} \mathrm{C}$ for $2 \mathrm{~h}$. In addition, all samples were prepared by the same method.

An appropriate content of OLDH was ultrasonically dispersed and mechanically stirred in an acetone solution $(1 \mathrm{~g} /$ $100 \mathrm{~mL}$ acetone) at room temperature for $0.5 \mathrm{~h}$. Then, the $\mathrm{EP}$ was added to the slurry and stirred at $80^{\circ} \mathrm{C}$ for $3 \mathrm{~h}$. The slurry was transferred to an oil bath and stirred at $130{ }^{\circ} \mathrm{C}$ for $0.5 \mathrm{~h}$ to evaporate the remaining acetone. A certain amount of DiDOPO was added to EP and stirred for $0.5 \mathrm{~h}$. Then, the system temperature was cooled to $50{ }^{\circ} \mathrm{C}$. The curing agent was introduced into the system to be mixed. The curing conditions were the same as those employed for the EP/DiDOPO composite. Fig. 1 shows the preparation of EP/DiDOPO/OLDH, and Table 1 lists the composition of the EP thermosets. To prepare the EP/ OLDH composites, the same procedure was followed.

\subsection{Characterization}

UL-94 measurement (CZF-2, Jiangning, China; dimensions of $130 \times 13 \times 3.2 \mathrm{~mm}^{3}$ ) was carried out according to ASTM D3801. LOI (according to ASTM D2863-77) was measured by a JF-3 oxygen index meter (Jiangning, China), with a sample size of
$100 \times 6.5 \times 3.2 \mathrm{~mm}^{3}$. The cone calorimeter test (CCT; FTT, UK) was conducted on the basis of ASTM E1354/ISO 5660. Specimens with dimensions of $100 \times 100 \times 6 \mathrm{~mm}^{3}$ and an external heat flux of $50 \mathrm{~kW} \mathrm{~m}^{-2}$ were selected; typically, the CCT data were replicable within $\pm 5 \%$. The thermal stability of all testing materials was evaluated by thermogravimetry (TG) using a TG 219 F3 thermal analyzer (Netzsch Instruments Co. Ltd., Germany) at a constant scanning rate of $10{ }^{\circ} \mathrm{C} \mathrm{min}^{-1}$ under nitrogen at temperatures ranging from 40 to $800{ }^{\circ} \mathrm{C}$. The microstructure of the carbon residue from the CCT of thermosets was observed on an FEI Quanta 250 FEG field-emission scanning electron microscope (SEM) (FEI Inc., U.S.A.) under high vacuum at a voltage of $20 \mathrm{kV}$. The Fourier transform infrared (FTIR) spectra of the LOI residues were recorded on a Nicolet is50-type spectrometer (Thermo Fisher Scientific Inc., U.S.A.). The LOI residue crown and initial pyrolysed residue samples were selected from specimens that self-extinguished within 2 min and exhibited burns initiating at less than $30 \mathrm{~mm}$ from the top. X-ray photoelectron spectroscopy (XPS) profiles of the char residue were recorded on a Thermo Escalab 250Xi system (Thermo Fisher Scientific Inc., U.S.A.) using $\mathrm{Al} \mathrm{K} \alpha$ excitation radiation $(h \nu=1253.6 \mathrm{eV})$. The TGFTIR instrument consisted of a TG219 F3 system (Netzsch Instruments Co., Ltd., Germany); an FTIR spectrometer (Nicolet IS50, Thermo Fisher Scientific Inc., U.S.A.); and a transfer tube with an inner diameter of $1 \mathrm{~mm}$ connected to the TG and IR cells. Measurements were carried out from 30 to $600{ }^{\circ} \mathrm{C}$ at a linear heating rate of $20{ }^{\circ} \mathrm{C} \min ^{-1}$ under a nitrogen flow of $30 \mathrm{~mL} \mathrm{~min}^{-1}$. Tensile and flexural measurements (Instron WDW100 tensile tester) were carried out at cross-head speeds of 50 and $2 \mathrm{~mm} \mathrm{~min}^{-1}$ at room temperature, respectively. The notched impact strength was evaluated on a ZBC1000 universal testing machine (Shenzhen Sans Co., Ltd., China). XRD patterns were recorded on a Bruker D8 Advance (Germany) XRD instrument equipped with a $\mathrm{Cu}-\mathrm{K} \alpha$ tube.

\section{Results and discussion}

\subsection{Flame retardancy of EP composites}

To verify the presence of a synergistic effect between DiDOPO and OLDH for improving the flame retardancy of EP, three groups (EP/DiDOPO1, EP/OLDH1, EP/DiDOPO0.5/OLDH0.5; $\mathrm{EP} /$ DiDOPO5, EP/OLDH5, EP/DiDOPO2.5/OLDH2.5; and EP/ DiDOPO10, EP/OLDH10, EP/DiDOPO5/OLDH5) were compared. The LOI and UL-94 vertical burning test rating was utilised as a qualitative method to evaluate the flame-retardant properties of polymeric materials. Hence, the LOI value and UL-94 rating of the EP composites are estimated (Table 1). Experimental results revealed that pure EP can be easily flammable with a low LOI of $21.8 \%$, but it did not pass the UL-94 test. Compared with that of pure EP, the LOI of the EP/DiDOPO composite increased to $38.0 \%$, and the V-0 rating was still observed with the incorporation of $10 \mathrm{wt} \%$ DiDOPO. The flame retardancy of the EP/DiDOPO composites was clearly improved effectively with a specific loading of DiDOPO. In the UL-94 test, except for the EP/DiDOPO1 composite, the EP/DiDOPO composite attained the V-0 rating because of the self-extinguishing phenomenon of DiDOPO. For $\mathrm{EP} / \mathrm{OLDH}$ systems, the addition of $1 \mathrm{wt} \%$ of OLDH effectively 

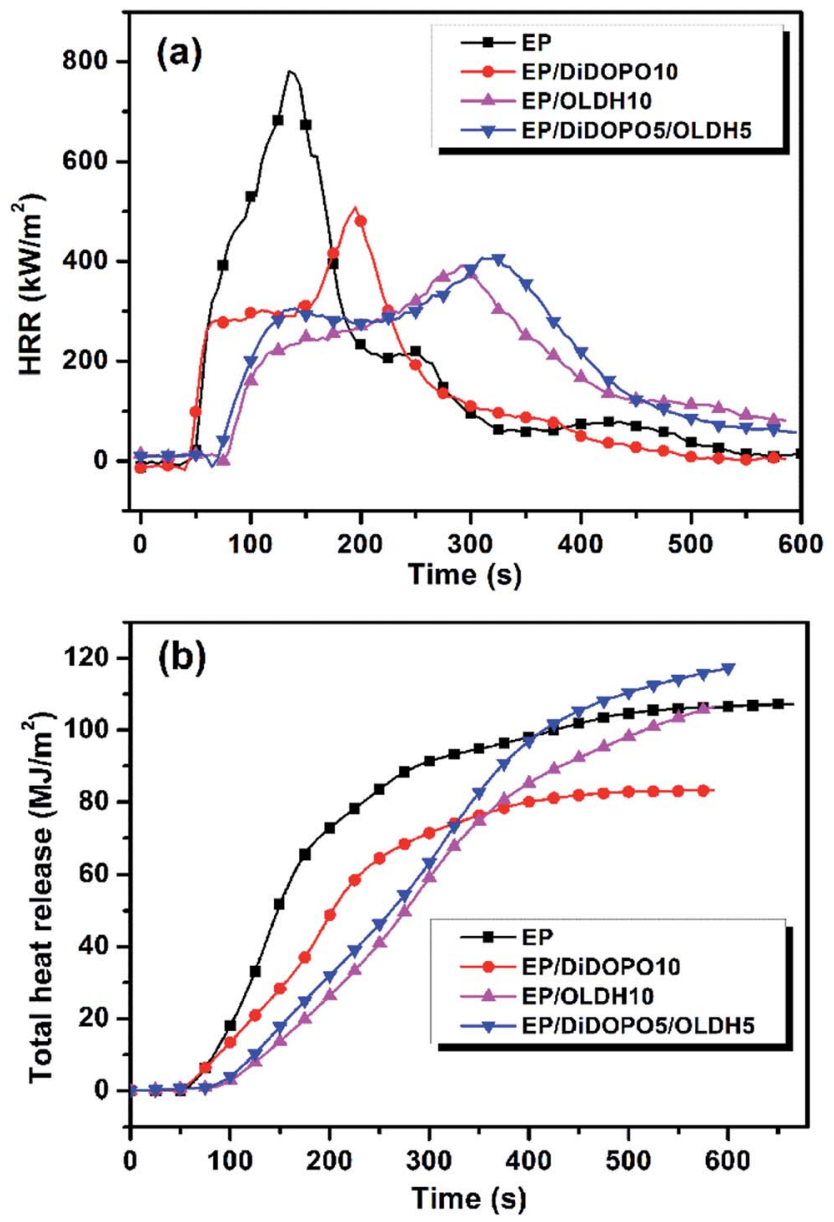

Fig. 2 (a) Heat release rate (HRR) and (b) total heat release (THR) curves for samples from the cone calorimeter test (CCT).

increased the LOI of EP, but the LOI was not further improved with increasing OLDH content. This result is in agreement with that reported by Liu et al. ${ }^{16}$ For EP/DiDOPO/OLDH systems, DiDOPO and OLDH exhibited a synergistic effect for improving the LOI of EP. The LOI was increased to $25.2 \%$ with the addition of $0.5 \mathrm{wt} \%$ DiDOPO and $0.5 \mathrm{wt} \% \mathrm{OLDH}$. The LOI is greater than that obtained with the addition of the same amount, i.e., $1 \mathrm{wt} \%$, of only DiDOPO (24.1\%) or OLDH (23.6\%). The EP/DiDOPO0.5/ OLDH0.5 composites exhibited a V-0-rating compared to EP/ DiDOPO0.5 (V-2-rating) and EP/OLDH (N.R.). Hence, the composites exhibited better flame-retardance properties with the addition of specific amounts of DiDOPO and OLDH.

To further investigate the flame-retardance properties of composites, parts of samples examined above were selected to investigate the fire behaviour by CCT. Fig. $2 \mathrm{a}$ and $\mathrm{b}$ and Table 2 summarise the results. Pure EP rapidly burned after ignition, and the heat release rate (HRR) sharply increased with a peak heat release rate (pHRR) of $781 \mathrm{~kW} \mathrm{~m}^{-2}$ at $135 \mathrm{~s}$; finally, the sample burns out within $600 \mathrm{~s}$. In contrast, the pHRR values of all the flame-retardant EP/DiDOPO, EP/OLDH, and EP/DiDOPO/ OLDH composites were considerably reduced. The EP/ DiDOPO5/OLDH5 composite exhibited a low pHRR of $406 \mathrm{~kW} \mathrm{~m}^{-2}$. This value corresponded to a decrease of $48.1 \%$ compared with that of pure EP. In addition, the pHRR of EP/ DiDOPO5/OLDH5 was decreased by $38.2 \%$ compared with that of EP/DiDOPO10. Notably, DiDOPO increased the LOI and improved the rating of the UL-94 test, but exhibited the minimal effect on the pHRR values in CCT for the flame EP composites. In addition, Long et $a{ }^{23}$ have reported a similar result. They stated that this phenomenon is related to the marginal yield of char obtained during combustion. In addition, Buczko et al. ${ }^{18}$ have proposed that DOPO derivatives mainly play a role in the flame-retardant mechanism of gas-phase inhibition and increased the melt-flow drip. Therefore, the data in LOI and UL94 tests of EP/DiDOPO are correlated to the gas-phase flame inhibition mechanism. The pHRR of EP/OLDH and $\mathrm{EP} /$ DiDOPO/OLDH increased, while the pHRR decreased in comparison with that of pure EP, related to a considerable amount of aqueous vapour generated by OLDH in combustion.

Fig. $2 \mathrm{~b}$ shows the THR curves of the materials. After burning, the THR of neat EP was $107 \mathrm{MJ} \mathrm{m}^{-2}$. Compared with that of pure $\mathrm{EP}$, those of the EP/DiDOPO10 composites decreased, indicating that the EP/DiDOPO composites effectively promote EP to construct a protective char layer during combustion. However, the THR of EP/OLDH10 increased in comparison with that of EP/DiDOPO5. With the addition of DiDOPO and OLDH,

Table 2 Data obtained from cone calorimeter test (CCT) of different samples ${ }^{a}$

\begin{tabular}{|c|c|c|c|c|c|c|}
\hline Samples & TTI (s) & pHRR $\left(\mathrm{kW} \mathrm{m}^{-2}\right)$ & $T_{\mathrm{Phrr}}(\mathrm{s})$ & THR $\left(\mathrm{MJ} \mathrm{m}^{-2}\right)$ & av-EHC $\left(\mathrm{MJ} \mathrm{kg}^{-1}\right)$ & $\operatorname{TSR}\left(\mathrm{m}^{2} \mathrm{~m}^{-2}\right)$ \\
\hline $\mathrm{EP}$ & 32 & 781 & 135 & 107 & 19 & 4250 \\
\hline EP/DiDOPO1 & 33 & 516 & 225 & 116 & 18 & 5199 \\
\hline EP/DiDOPO10 & 38 & 508 & 195 & 83 & 13 & 5559 \\
\hline EP/OLDH1 & 35 & 543 & 270 & 121 & 21 & 3615 \\
\hline EP/OLDH5 & 35 & 521 & 225 & 104 & 22 & 3204 \\
\hline EP/DiDOPO2.5/OLDH2.5 & 44 & 420 & 300 & 120 & 18 & 4446 \\
\hline EP/DiDOPO5/OLDH5 & 46 & 406 & 310 & 82 & 15 & 3685 \\
\hline
\end{tabular}

${ }^{a}$ TTI: time to ignition; pHRR: peak heat release rate; TpHRR: time to peak heat release rate; THR: total heat release; av-EHC: average effective heat of combustion; TSR: total smoke rate. 
the THR of EP/DiDOPO/OLDH was considerably less than those of pure EP, ER/DiDOPO, and EP/OLDH composites. A synergistic effect was observed between DiDOPO and OLDH to decrease the THR of EP. The THR of EP decreased from $107 \mathrm{MJ} \mathrm{m}^{-2}$ to $82 \mathrm{MJ} \mathrm{m}^{-2}$ with the addition of $5 \mathrm{wt} \%$ DiDOPO and $5 \mathrm{wt} \% \mathrm{OLDH}$ together. This THR is less than that observed for DiDOPO (83 $\mathrm{MJ} \mathrm{m}^{-2}$ ) or OLDH $\left(106 \mathrm{MJ} \mathrm{m}^{-2}\right.$ ) alone at the same addition level, $10 \mathrm{wt} \%$.

Table 2 summarises the parameters obtained from CCT, including the time to ignition (TTI), total smoke rate (TSR), and the average effective heat of combustion (av-EHC). The TSR analysis was combined with the effective heat of combustion (EHC). A high TSR accompanied by a low av-EHC value indicates the formation of more non-combustible components in the gas phase. The mean EHC of pure EP decreased in comparison with those of EP/DiDOPO and EP/DiDOPO/OLDH (Table 2). In all of the composites, the av-EHC values of EP/DiDOPO and EP/ DiDOPO/OLDH clearly decreased in comparison with that of neat EP. The clear decrease in the av-EHC is possibly related to the possible release of the $\mathrm{PO}$ and phenoxyl free radicals. The TSR of pure EP was $4250 \mathrm{~m}^{2} \mathrm{~m}^{-2}$, whereas those of the EP/ $\mathrm{OLDH}$ composites were considerably decreased. Moreover, that of the EP/DiDOPO/OLDH composites increased, implying
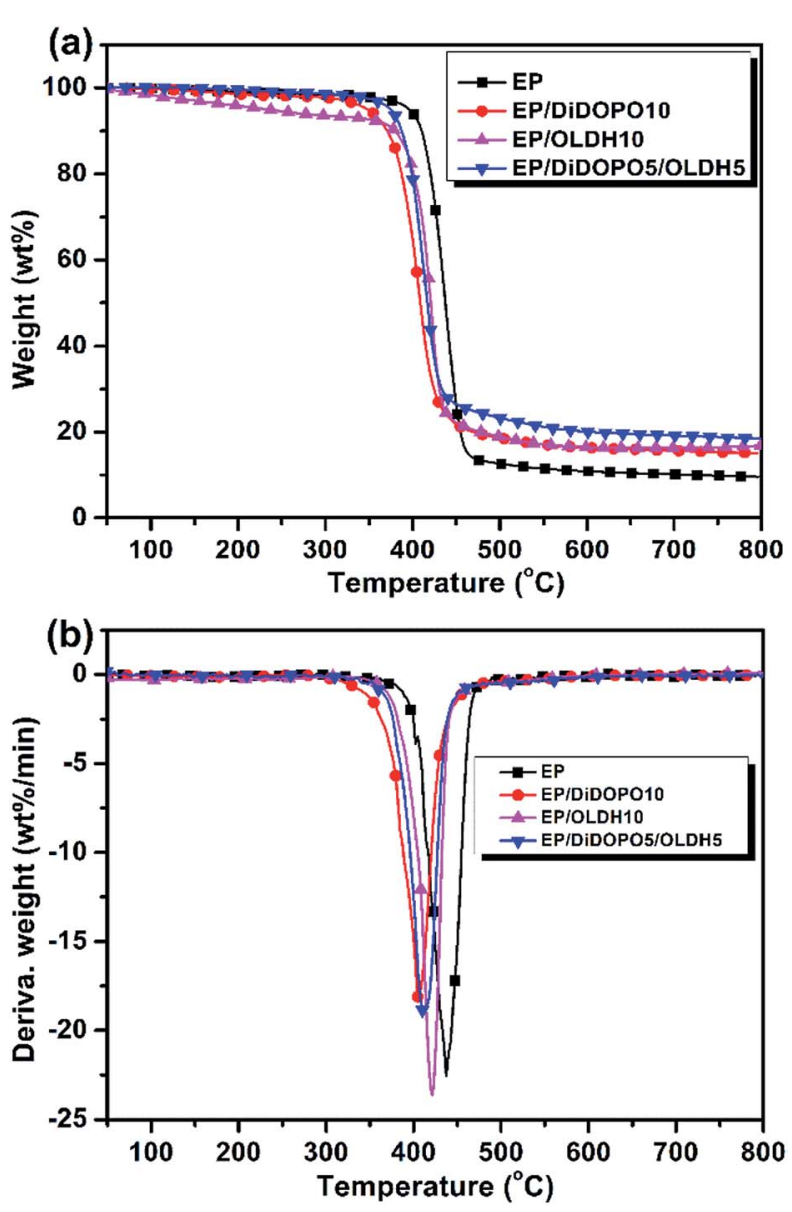

Fig. 3 (a) Thermogravimetry (TG) and (b) differential thermogravimetry (DTG) curves of the samples under $\mathrm{N}_{2}$ at a heating rate of $10{ }^{\circ} \mathrm{C} \mathrm{min}^{-1}$.
Table 3 TG and DTG data for different samples under $\mathrm{N}_{2}$

\begin{tabular}{|c|c|c|c|c|c|}
\hline \multirow[b]{2}{*}{ Samples } & \multirow{2}{*}{$\begin{array}{l}T_{\text {initial }} \\
\left({ }^{\circ} \mathrm{C}\right)\end{array}$} & \multirow{2}{*}{$\begin{array}{l}T_{\max } \\
\left({ }^{\circ} \mathrm{C}\right)\end{array}$} & \multirow{2}{*}{$\begin{array}{l}\text { Rate of } T_{\max } \\
\left(\mathrm{wt} \% \min ^{-1}\right)\end{array}$} & \multicolumn{2}{|c|}{$\begin{array}{l}\text { Char residue } \\
\text { (wt\%) }\end{array}$} \\
\hline & & & & $700{ }^{\circ} \mathrm{C}$ & $800{ }^{\circ} \mathrm{C}$ \\
\hline EP & 396 & 43 & 22.6 & 10.1 & 9.7 \\
\hline EP/DiDOPO10 & 349 & 40 & 18.1 & 15.6 & 15.3 \\
\hline EP/OLDH10 & 231 & 42 & 23.4 & 16.3 & 16.8 \\
\hline $\begin{array}{l}\text { EP/DiDOPO5/ } \\
\text { OLDH5 }\end{array}$ & 374 & 41 & 19.0 & 19.1 & 18.4 \\
\hline
\end{tabular}

that more organic structures in the EP not only participate in carbonization but also are retained in the condensed phase instead to being converted to fuel in the gas phase. ${ }^{13}$

\subsection{Thermal stability of EP composites}

Fig. 3a and b shows the TG and differential thermogravimetry (DTG) curves for EP and its flame-retardant composites under nitrogen, respectively.

The onset degradation temperature $\left(T_{\text {initial }}\right)$ of the samples was evaluated by the temperature at the $5 \mathrm{wt} \%$ weight loss. Solid residues were obtained at $700{ }^{\circ} \mathrm{C}$ and $800{ }^{\circ} \mathrm{C}$ from the TG curves. The temperature at the maximum weight loss rate $\left(T_{\max }\right)$ of the samples was obtained from the DTG curves. Table 3 summarises the data obtained. Fig. 2 shows the decomposition processes of all samples, which mainly occurred between $350{ }^{\circ} \mathrm{C}$ and $500{ }^{\circ} \mathrm{C}$, with one distinguishable weight loss maximum. The onset degradation temperature $\left(T_{\text {initial }}\right)$ for EP was $396{ }^{\circ} \mathrm{C}$; the highest mass loss rate at $T_{\max }$ was observed at $22.6 \mathrm{wt} \% \mathrm{~min}^{-1}$ at $437^{\circ} \mathrm{C}$; and a char yield of $9.7 \mathrm{wt} \%$ was obtained at $800^{\circ} \mathrm{C}$ under $\mathrm{N}_{2}$. All of the epoxy composites exhibited a similar one-stage degradation process. The $T_{\text {initial }}$ for the EP/DiDOPO10, EP/

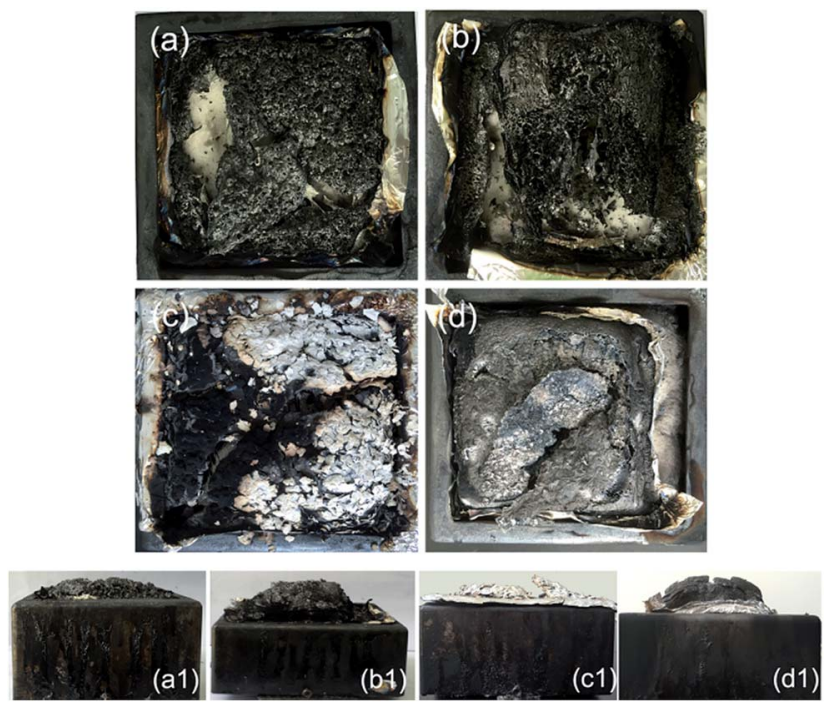

Fig. 4 Top view of the char residues after the cone calorimeter tests of (a) EP, (b) EP/DiDOPO10, (c) EP/OLDH10, and (d) EP/DiDOPO5/ OLDH5 composites. (a1)-(d1) shows the front views of EP, EP/ DiDOPO10, EP/OLDH10, and EP/DiDOPO5/OLDH5 composites. 
OLDH10, and EP/DiDOPO5/OLDH5 composites decreased to 349, 231, and $374{ }^{\circ} \mathrm{C}$, respectively. Moreover, the incorporation of $\mathrm{OLDH}$ led to an increase in the char yield at $800{ }^{\circ} \mathrm{C}$. The solid residue of the EP composites increased to 15.3, 16.8, and $18.4 \mathrm{wt} \%$ at $800{ }^{\circ} \mathrm{C}$, respectively, with the addition of $10 \mathrm{wt} \%$ DiDOPO, 10 wt $\%$ OLDH, 5 wt $\%$ DiDOPO, and 5 wt\% OLDH. With the introduction of DiDOPO and OLDH, the mass loss rates at $T_{\max }$ decreased further, suggesting that the introduction of DiDOPO leads to a considerable decrease in $T_{\text {initial }}$.

The decrease in $T_{\text {initial}}$, the mass loss rate at $T_{\max }$, and the residue weight notably increased, suggesting that lowtemperature decomposition accelerates high-temperature char formation and increases the amount of char residue.

\subsection{Morphology analysis of the residual chars after CCT}

Besides the aforementioned flame-retardant effect derived from the gas-phase activity and charring, the protective barrier generated from the intumescent char layer also imparted better fire resistance to the epoxy thermoset. SEM and visual observation were employed to examine the morphology of the char residues. Fig. 4 and 5 show the digital photographs and SEM images of the char residues from the CCT, respectively. Pure EP clearly decreased after combustion (Fig. 4). However, with the introduction of DiDOPO, the structure of char layers became dense and with distinct intumescent char. The slow heat release of EP/OLDH10 was justified by the formation of a compact residue. This structure was brittle, albeit with good mechanical strength, integrity, coherence, and adherence to the substrate. The shell comprised a bilayer structure, i.e., a white, porous internal face (formed by metal oxides) and a compact black external face (formed by carbonaceous residues); some of the carbonaceous residues may originate from the soot formed in the gas phase. ${ }^{34}$ On the other hand, the EP/DiDOPO5/OLDH5 surface, after the CCT, was completely fragmented and
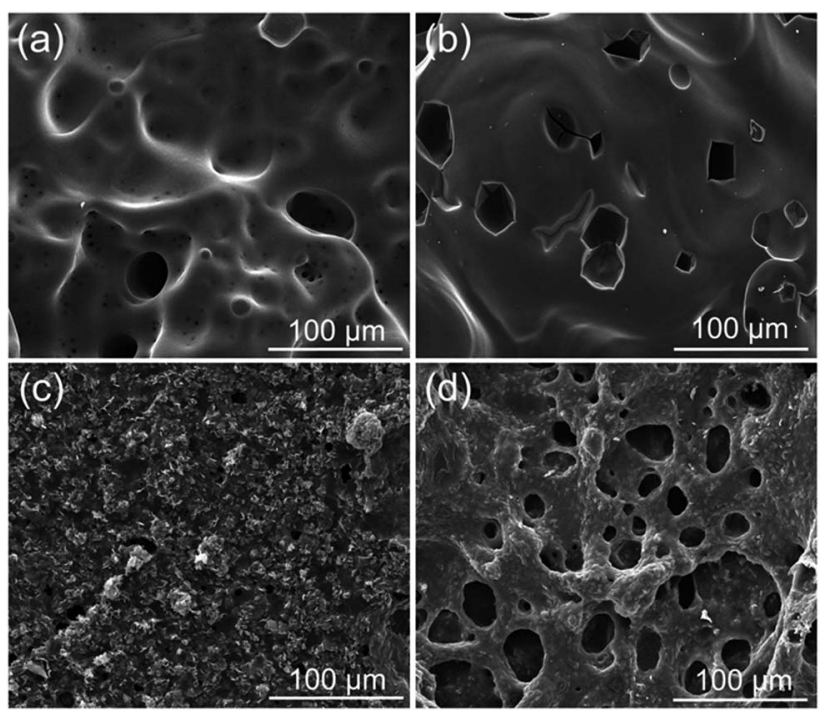

Fig. 5 SEM images of the char residues after the cone calorimeter tests of (a) EP, (b) EP/DiDOPO10, (c) EP/OLDH10, and (d) EP/ DiDOPO5/OLDH5 composites. exhibited intumescent behaviour, as well as a thick shielding layer. Therefore, from the front views of the char layers, the EP/ DiDOPO/OLDH composites catalyse the formation of a compact char layer, preventing the further decomposition of the epoxy matrix.

Fig. 5 shows the morphologies of the EP, EP/DiDOPO10, EP/ OLDH10, and EP/DiDOPO5/OLDH5 residues after CCT. The residue of EP exhibited a badly broken carbon layer structure, verifying the failure to prevent the flame and heat transfer. The residue of EP/DiDOPO10 became denser, and the holes were reduced compared with that of pure EP and exhibited a thick, compact char layer; this char layer surface was covered with the decomposition product of the phosphorus-containing group. ${ }^{35}$ In EP/OLDH10, some holes existed in the slightly rugged char residue. In $\mathrm{EP} / \mathrm{DiDOPO5/OLDH5}$, the morphology of char residues was similar to that of EP/DiDOPO, and some cracks were observed, implying that the barrier properties are inferior to EP/ DiDOPO and EP/OLDH. Combining the results obtained from the CCT and TG tests, the macro and micro-morphologies of the char residue well demonstrated the gas- and condensed-phase working modes during the burning of the EP/DiDOPO/OLDH composites.

\subsection{Chemical analysis of the residual chars after the cone calorimeter and LOI tests}

To investigate the flame-retardant mechanism, TG-IR was employed to analyse the gas products of the resins during thermal degradation under nitrogen. Fig. 6 and 7 shows the 3D TG-FTIR and FTIR spectra of the gas phase during the thermal degradation of EP, EP/DiDOPO10, EP/OLDH10, and EP/ DiDOPO5/OLDH5, respectively. The main products from the thermal decomposition of EP comprised compounds containing $-\mathrm{OH}$ (e.g., $\mathrm{H}_{2} \mathrm{O}$ and phenol; 3500-3600 $\left.\mathrm{cm}^{-1}\right) ; \mathrm{CO}_{2}$ $\left(2358 \mathrm{~cm}^{-1}\right)$; CO $\left(2180 \mathrm{~cm}^{-1}\right)$; aromatic ethers $\left(1261 \mathrm{~cm}^{-1}\right)$; hydrocarbons (C-H stretching at $\left.1176 \mathrm{~cm}^{-1}\right)$; compounds containing aromatic rings (1605 and $\left.1510 \mathrm{~cm}^{-1}\right)$; and $\mathrm{C}_{\mathrm{Ar}}-\mathrm{H}$
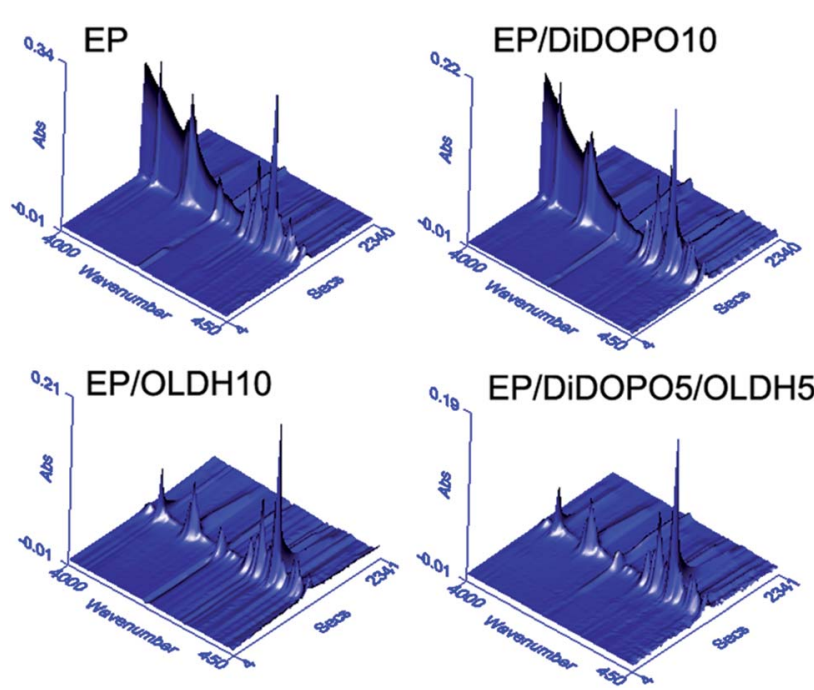

Fig. 6 3D TG-FTIR profiles for the pyrolysis products obtained for EP, EP/DiDOPO10, EP/OLDH10, and EP/DiDOPO5/OLDH5 composites. 

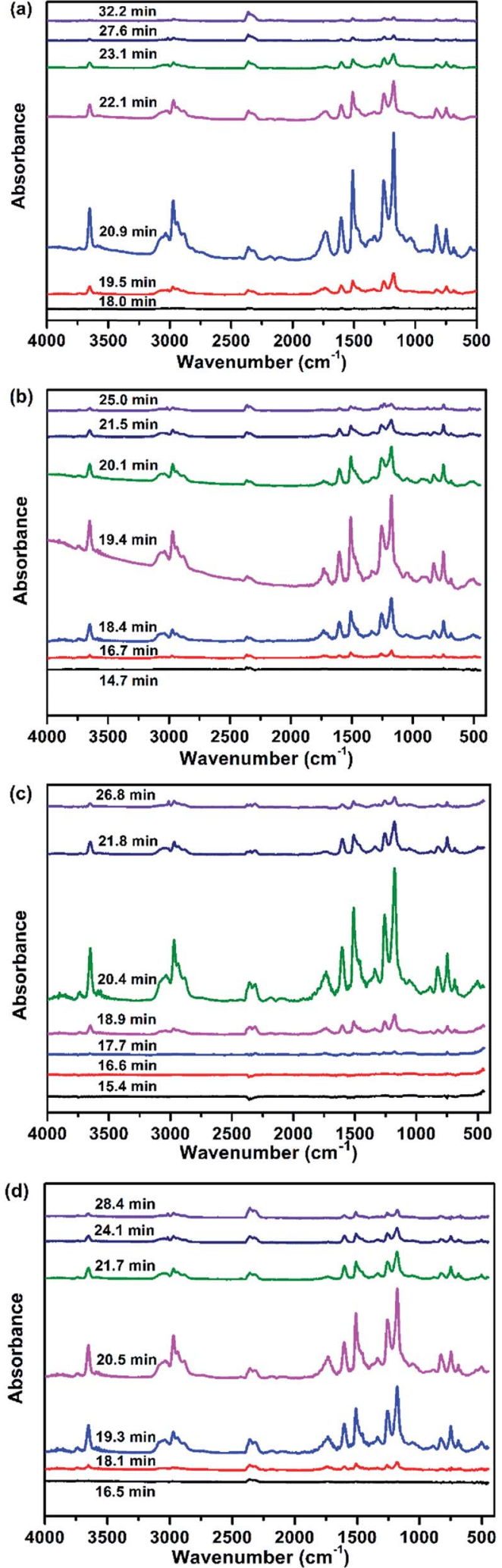

Fig. 7 FTIR spectra of the products obtained from the pyrolysis of the EP composites at different times: (a) EP, (b) EP/DiDOPO10, (c) EP/ OLDH10, and (d) EP/DiDOPO5/OLDH5. $\left(3035 \mathrm{~cm}^{-1}, 827 \mathrm{~cm}^{-1}\right.$, and $\left.744 \mathrm{~cm}^{-1}\right) \cdot{ }^{36-38}$ Depolymerisation is well known to be the main process related to the thermal degradation of polymers. During depolymerisation, the main decomposition products were $\mathrm{CO}_{2}$, phenol, and hydrocarbons.

From the distinct pyrolysis products of the composites during thermal degradation, the depolymerisation of EP and EP composites is considerably different. Pure EP dramatically decomposed because of the absence of additives. The products included high amounts of carboxylic acid and aliphatic hydrocarbons during decarboxylation and dissociation of the main chains. On the contrary, the decomposition of EP/DiDOPO10, EP/OLDH10, and EP/DiDOPO5/OLDH5 was considerably slower. The reasons will be discussed as follows.

Fig. 7 shows the analysis of the gases evolved for EP/ DiDOPO10, EP/OLDH10, and EP/DiDOPO5/OLDH5 at different times. With increasing time, the EP/DiDOPO10 composites exhibited characteristic bands of $-\mathrm{O}-\mathrm{H}\left(3652 \mathrm{~cm}^{-1}\right) ; \mathrm{C}_{\mathrm{Ar}}-\mathrm{H}$ (3035 $\mathrm{cm}^{-1}, 827 \mathrm{~cm}^{-1}$, and $\left.744 \mathrm{~cm}^{-1}\right)$; C-H $\left(2972 \mathrm{~cm}^{-1}\right.$ and $\left.1339 \mathrm{~cm}^{-1}\right) ; \mathrm{CO}_{2}\left(2358 \mathrm{~cm}^{-1}\right)$; $\mathrm{CO}\left(2180 \mathrm{~cm}^{-1}\right)$; hydrocarbons ( $-\mathrm{CH}_{3}$ and $-\mathrm{CH}_{2}-\mathrm{S}$ groups: $2950-2850$ and $1100-1300 \mathrm{~cm}^{-1}$ ); and compounds containing aromatic rings (1605 and $\left.1510 \mathrm{~cm}^{-1}\right),,^{38-40}$ which is similar to that of EP. An absorption band was observed at $1261 \mathrm{~cm}^{-1}(\mathrm{P}=\mathrm{O})$, which almost coincided with the characteristic peaks of the EP matrix. Hence, the polyphosphate structures are formed by the decomposition of DiDOPO. These polyphosphate structures can react with other pyrolysis products (such as phenol or bisphenol) to form the P$\mathrm{O}-\mathrm{Ph}$ bond. ${ }^{23}$ The formation of these compounds is mainly related to the decomposition of the EP.

In the EP/OLDH10 composite, the change in the degradation corresponded to the loss of interlayer water from $\mathrm{LDH}$ at low temperature. At high temperature, the change of degradation corresponded to the condensation of hydroxyl groups from the octahedral layer, in addition to the decomposition of the anion $\mathrm{NO}_{3}{ }^{2-}$. These two decomposition stages were strongly endothermic. ${ }^{16} \mathrm{EP} / \mathrm{OLDH} 10$ released more water than pure EP at temperatures less than $500{ }^{\circ} \mathrm{C}$ because of the release of $\mathrm{H}_{2} \mathrm{O}$,

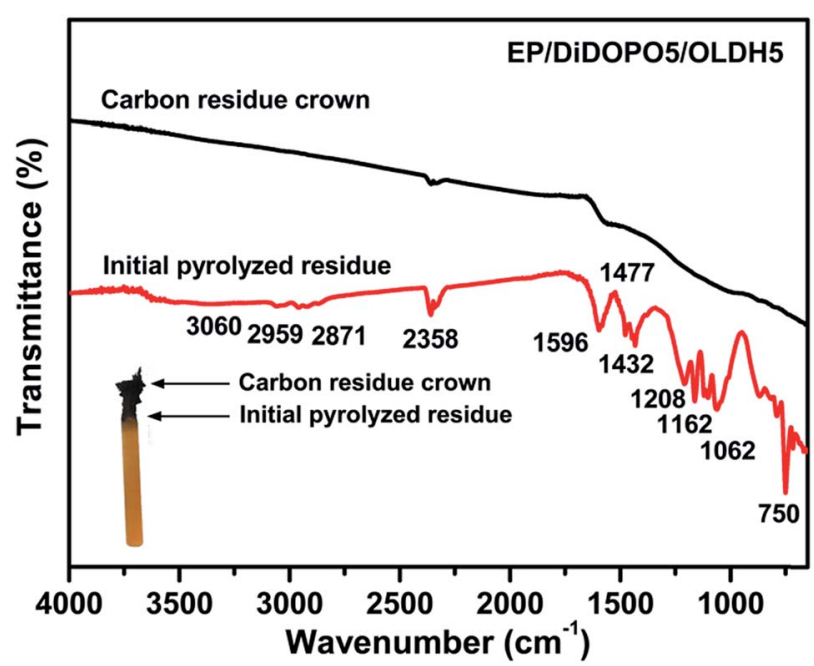

Fig. 8 FTIR spectra of the residual char for the EP/DiDOPO5/OLDH5 composites after the LOI test. 
which can be rationalised by the following reasons. The OLDH interlayer structure loaded substantial water for the composites. The - $\mathrm{OH}$ groups present in OLDH can react with the carboxylic acid formed by the decomposition of EP to release more water. ${ }^{24}$ Between 350 and $450{ }^{\circ} \mathrm{C}$, the gas products of EP/OLDH10 were the same as those obtained for EP, except for the disappearance of the aromatic ring. The gas products of EP/DiDOPO/OLDH were basically the same as those observed for EP.

The results also implied that most of the decomposition products of $\mathrm{EP} / \mathrm{DiDOPO} / \mathrm{OLDH}$ composites remain in the condensed phase. Moreover, lower $T_{\text {initial }}$ and $T_{\max }$ values were observed for the EP/OLDH and EP/DiDOPO/OLDH composites, although the pyrolysis products of EP and EP/DiDOPO/OLDH were similar. Hence, DiDOPO and OLDH can promote the degradation of EP at a low temperature.

FTIR was also employed to examine the char residue obtained from the LOI test. The matrix was burned to form a char crown, and the absorption peaks of the residue completely differed from those of the previous matrix (Fig. 8). The spectrum of the char crown was not obvious. During the initial pyrolysis stage, the absorption bands at $2959 \mathrm{~cm}^{-1}$ and $2871 \mathrm{~cm}^{-1}$ were related to the saturated $-\mathrm{CH}_{2}$ - and $\mathrm{C}-\mathrm{H}$ stretching, respectively. Vibration bands characteristic of benzene (C-C stretching at $1432 \mathrm{~cm}^{-1}$ and $\mathrm{C}-\mathrm{H}$ in-plane bending at $1162 \mathrm{~cm}^{-1}$ ). A sharp peak corresponding to the aromatic ring $\mathrm{C}=\mathrm{C}$ was observed at $1596 \mathrm{~cm}^{-1}$. Characteristic peaks observed at 750 and $1062 \mathrm{~cm}^{-1}$

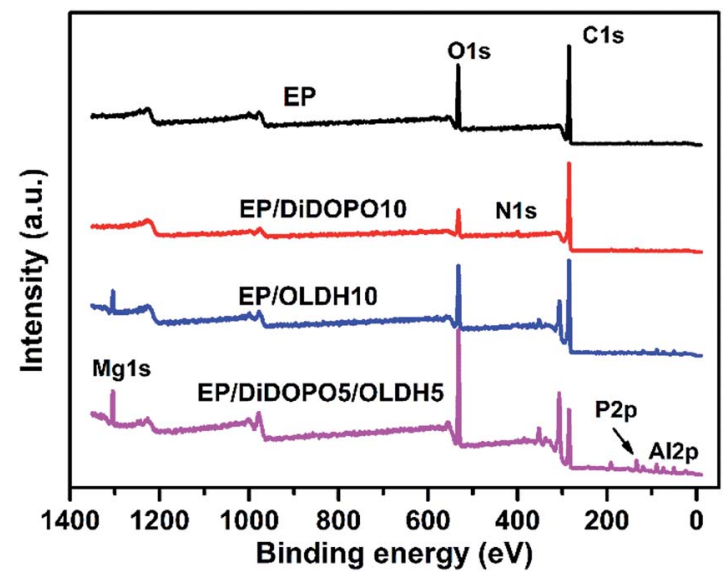

Fig. 9 XPS spectra of the char residue on EP composites. were related to the $\mathrm{P}-\mathrm{O}-\mathrm{P}$ and $\mathrm{P}-\mathrm{O}-\mathrm{C}$ bonding, respectively. ${ }^{\mathbf{8 4 1}}$ The results suggested the formation of phosphate compounds.

XPS was employed to examine the chemical components of the residual char for EP, EP/DiDOPO10, EP/OLDH10, and EP/ DiDOPO5/OLDH5 composites after CCT. Three bands were observed from the $\mathrm{C}$ 1s spectra. A peak at around $284.8 \mathrm{eV}$ corresponded to the $\mathrm{C}-\mathrm{H}$ and $\mathrm{C}-\mathrm{C}$ in aliphatic and aromatic species (Fig. 9 and Table 4). The peak centred at $532.7 \mathrm{eV}$ corresponded to $-\mathrm{O}-$ in $\mathrm{C}-\mathrm{O}-\mathrm{C}, \mathrm{C}-\mathrm{O}-\mathrm{P}$, and/or $\mathrm{C}-\mathrm{OH}$ groups. ${ }^{42}$ The peak at approximately $400.0 \mathrm{eV}$, as observed for the EP and EP composites, was related to the $\mathrm{N} 1 \mathrm{~s}$ of the oxidised nitrogen compounds in the residual char. The single peak between 133 and $135 \mathrm{eV}$ in the $\mathrm{P} 2 \mathrm{p}$ spectrum corresponded to pyrophosphate and/or polyphosphate. ${ }^{\mathbf{4 3 , 4 4}}$

In the XPS spectrum of the EP/OLDH and EP/DiDOPO/OLDH composites, some peaks were observed at around $74 \mathrm{eV}(\mathrm{Al} 2 \mathrm{p})$ and $1304 \mathrm{eV}(\mathrm{Mg} 2 \mathrm{p})$. The weight ratios of magnesium and aluminum for EP/OLDH and EP/DiDOPO/OLDH were $4.0 \%$ and $6.3 \%$ and $2.6 \%$ and $5.2 \%$, respectively. The addition of a flame retardant considerably affected the flame retardancy and thermal degradation of the composites (Table 4). The atom percentages of carbon in the char residues of EP and EP/ DiDOPO10 were $78.7 \%$ and $82.0 \%$, respectively. The atom percentage of oxygen in the char residues of EP/DiDOPO10 was less than that in the char residue of EP, indicating that the incorporation of DiDOPO into EP can promote the formation of char residues and enhance the thermal oxidative resistance of the EP matrix. At high temperatures, DiDOPO can remarkably increase the char yield and improve the thermal oxidative stability of the char layer. With the addition of DiDOPO and $\mathrm{OLDH}$, the content of $\mathrm{Mg}$ and $\mathrm{Al}$ was considerably greater than that of EP/OLDH10. Hence, the nanofillers are speculated to be transferred to the matrix surface during combustion, which can form an effective char layer and hence delay the permeation of heat and toxic gases. Moreover, the char layer was formed on the surface of the burning polymer, which insulated the heat transformation and the dispersion of oxygen into the underlying polymeric substrate.

Fig. 10 shows the schematic of the flame-retardant effect of DiDOPO and OLDH in the EP composites. During combustion, EP was degraded and released as combustible gas, and heat passed through the matrix without hindrance. EP formed some flammable drips and discontinuous char. In the EP/DiDOPO composite, DiDOPO released the PO', which trapped the $\mathrm{H}$

Table 4 XPS results of the residual char of EP composites

\begin{tabular}{|c|c|c|c|c|c|c|c|c|}
\hline & \multicolumn{2}{|l|}{ EP } & \multicolumn{2}{|c|}{ EP/DiDOPO10 } & \multicolumn{2}{|c|}{ EP/OLDH10 } & \multicolumn{2}{|c|}{ EP/DiDOPO5/OLDH5 } \\
\hline O 1s & 532.7 & 20.6 & 532.5 & 13.6 & 532.4 & 23.2 & 532.2 & 37.7 \\
\hline N 1s & 403.7 & 0.7 & 400.7 & 3.0 & 400.0 & 1.8 & 400.8 & 0.6 \\
\hline P $2 p$ & - & - & 133.6 & 1.3 & - & - & 134.1 & 5.0 \\
\hline
\end{tabular}


(a)
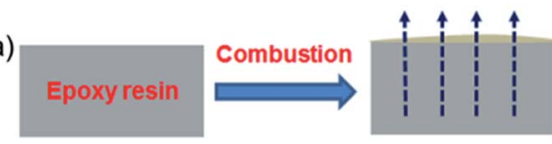

Gas phase

Condensed

phase

(b)

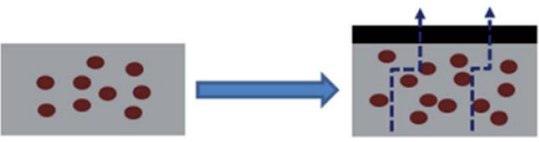

(c)

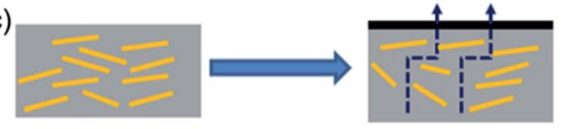

(d)

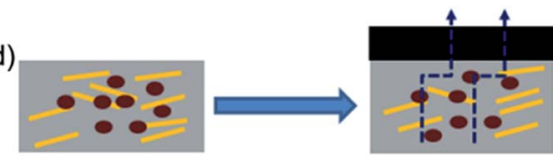

- DiDOPO OLDH

Gas transmission path

Fig. 10 Schematic of the flame-retardant mechanism of (a) EP, (b) EP/ DiDOPO, (c) EP/OLDH, and (d) EP/DiDOPO/OLDH composites.

and $\mathrm{OH}$ radicals in the gas phase and retarded the degradation and combustion of the polymer. Meanwhile, the pyrolysis products of the acid source from DiDOPO catalysed the formation of carbonaceous char, which protected the EP from further burning. ${ }^{45}$ In the EP/OLDH composite, the quality of the char residue was well correlated to the dispersion state of the nanofiller in the polymer matrix. The OLDHs were well dispersed, leading to a compact, continuous residue, which can simultaneously serve as an excellent insulator (protecting the underlying matrix from destruction by the irradiation of exterior heat) and mass transport barrier (which inhibits the escape of the flammable gases from the interior). ${ }^{46}$ Meanwhile, some OLDH could form aggregated stacks in the epoxy matrix; thus, the residue exhibits a cracked surface without sufficient cohesion. The degraded volatiles can freely escape from these cracks to feed the flame; meanwhile, the irradiated heat easily penetrates through these gaps to consume the underlying polymer matrix. These results corresponded well with the considerably higher PHRR and lower values of LOI for EP/OLDH. In the EP/ DiDOPO/OLDH composite, the improvement can be classified into two phases, condensed phase and gas phase, respectively. OLDH was mainly responsible for the flame-retardancy effect in the condensed phase, while DiDOPO was mainly responsible for the condensed and gas phases. The effect of DiDOPO to capture the free radicals further retarded combustion.

The combination of DiDOPO and OLDH formed more continuous char in the EP matrix. The char extended the transformation path for heat and gas and retarded the degradation of EP.

\subsection{Mechanical properties of the EP composites}

Table $\mathrm{S} 1 \dagger$ summarises the mechanical properties of the EP composites. The tensile, flexural, and Izod notched impact strengths for the cured EP were $43.8 \mathrm{MPa}$, 70.6 $\mathrm{MPa}$, and $7.0 \mathrm{~kJ} \mathrm{~m}^{-2}$, respectively. However, the tensile strength slightly decreased to $34.6 \mathrm{MPa}$ and $42.9 \mathrm{MPa}$ with the addition of 10 wt\% DiDOPO and OLDH, respectively. Meanwhile, the tensile strength of EP/DiDOPO5/OLDH5 (61.5 MPa) increased by $40.4 \%$ compared with that of pure EP. Moreover, the flexural strength of $\mathrm{EP} / \mathrm{DiDOPO} / \mathrm{OLDH}$ increased by $25.2 \%$ with the incorporation of $5 \mathrm{wt} \%$ DiDOPO and OLDH into the epoxy resin. The Izod notched impact strength of the thermosets decreased with the increased content of the flame retardant. The elongation-at-break of the EP composites exhibited minimal change with the increase in the DiDOPO concentration from 1 to $5 \mathrm{wt} \%$. Thus, the limited deterioration in the mechanical performance of EP caused by DiDOPO and OLDH was probably related to the low addition content and rigidity derived from the aromatic group and even the uniform dispersion of DiDOPO and OLDH in the EP matrix. ${ }^{30}$

\section{Conclusions}

In this study, a phenethyl-bridged DOPO derivative (DiDOPO) and OLDH were used to prepare flame-retardant EP composites. The morphology, flame retardance, thermal stability, flameretardant mechanism, and mechanical properties of the EP composites were investigated. The UL-94 V-0 rating and an LOI of $38 \%$ were obtained with $10 \mathrm{wt} \%$ DiDOPO. An LOI of $22.1 \%$ was obtained for $\mathrm{EP} / \mathrm{OLDH}$ with $10 \mathrm{wt} \%$ of OLDH. A synergistic effect was observed between DiDOPO and OLDH to improve the LOI value of EP. The LOI value increased from 21.8 to 25.2 with the addition of $0.5 \mathrm{wt} \%$ of DiDOPO and $0.5 \mathrm{wt} \%$ of OLDH. This LOI was greater than that obtained with the addition of $1 \mathrm{wt} \%$ of DiDOPO or $1 \mathrm{wt} \%$ of OLDH alone. The simultaneous addition of DiDOPO and OLDH to the EP matrix decreased the pHRR from 781 to $406 \mathrm{KW} \mathrm{m}{ }^{-2}$ with the addition of $5 \mathrm{wt} \%$ DiDOPO and $5 \mathrm{wt} \%$ OLDH. In addition, SEM observation after CCT suggested that the char residue for the EP/DiDOPO/OLDH composite is considerably more continuous and compact than that of the OLDH- and DiDOPO-containing composites. Moreover, the chemical analysis of residual chars revealed that flame fillers are transferred to the matrix surface during combustion, which can form an effective char layer and delay the permeation of heat and toxic gases. Meanwhile, DiDOPO and OLDH were well dispersed in the matrix-reinforced EP composites. The good mechanical performance indicated that the flame-retardant EP composites exhibit potential for several applications. Hence, DiDOPO and OLDHs exert gas- and condensed-phase flameretardant effects.

\section{Conflicts of interest}

There are no conflicts to declare.

\section{Acknowledgements}

The research was supported by the Guizhou Science and Technology Cooperation Project (20157304) and the Guizhou Provincial Department of Education Natural Foundation (2015400). 


\section{References}

1 H. Huang, K. Zhang, J. Jiang, J. Li and Y. Liu, Polym. Int., 2017, 66, 85-91.

2 H. Gu, C. Ma, J. Gu, J. Guo, X. Yan, J. Huang, Q. Zhang and Z. Guo, J. Mater. Chem. C, 2016, 4, 5890-5906.

3 X. Zhao, H. V. Babu, J. Llorca and D.-Y. Wang, RSC Adv., 2016, 6, 59226-59236.

4 R. Jian, P. Wang, W. Duan, J. Wang, X. Zheng and J. Weng, Ind. Eng. Chem. Res., 2016, 55, 11520-11527.

5 S. Huo, J. Wang, S. Yang, J. Wang, B. Zhang, B. Zhang, X. Chen and Y. Tang, Polym. Degrad. Stab., 2016, 131, 106113.

6 S. Yang, J. Wang, S. Huo, M. Wang and L. Cheng, Ind. Eng. Chem. Res., 2015, 54, 7777-7786.

7 S. Liu, H. Yan, Z.-P. Fang and H. Wang, Compos. Sci. Technol., 2014, 90, 40-47.

8 G. Wang and Z. Nie, Polym. Degrad. Stab., 2016, 130, 143-154.

9 A. Wirasaputra, X. Yao, Y. Zhu, S. Liu, Y. Yuan, J. Zhao and Y. Fu, Macromol. Mater. Eng., 2016, 301, 982-991.

10 P. Wang and Z. Cai, Polym. Degrad. Stab., 2017, 137, 138-150.

11 J. J. Chruściel and E. Leśniak, Prog. Polym. Sci., 2015, 41, 67121.

12 X. Qian, L. Song, Y. Hu and R. K. K. Yuen, Thermochim. Acta, 2013, 552, 87-97.

13 E. N. Kalali, X. Wang and D.-Y. Wang, J. Mater. Chem. A, 2015, 3, 6819-6826.

14 W. Wang, H. Pan, Y. Shi, Y. Pan, W. Yang, K. M. Liew, L. Song and Y. Hu, Composites, Part A, 2016, 80, 259-269.

15 X. Wang, E. N. Kalali and D.-Y. Wang, ACS Sustainable Chem. Eng., 2015, 3, 3281-3290.

16 S. Liu, H. Yan, Z.-P. Fang, Z. Guo and H. Wang, RSC Adv., 2014, 4, 18652-18659.

17 X. Wang, W. Xing, X. Feng, B. Yu, L. Song and Y. Hu, Polym. Chem., 2014, 5, 1145-1154.

18 A. Buczko, T. Stelzig, L. Bommer, D. Rentsch, M. Heneczkowski and S. Gaan, Polym. Degrad. Stab., 2014, 107, 158-165.

19 X. Qian, L. Song, S. Jiang, G. Tang, W. Xing, B. Wang, Y. Hu and R. K. K. Yuen, Ind. Eng. Chem. Res., 2013, 52, 7307-7315.

20 S. Tang, L. Qian, X. Liu and Y. Dong, Polym. Degrad. Stab., 2016, 133, 350-357.

21 Y. L. Angell, K. M. White, S. E. Angell and A. G. Mack, U.S. Pat., US20120053265 A1, 2012.

22 Y. Qiu, L. Qian and W. Xi, RSC Adv., 2016, 6, 56018-56027.

23 L.-J. Long, J. Yin, W.-T. He, S.-H. Qin and J. Yu, Ind. Eng. Chem. Res., 2016, 55, 10803-10812.

24 Y. Qian, S. J. Zhou and X. L. Chen, Polym. Adv. Technol., 2017, 28, 353-361.
25 P. K. Kaul, A. J. Samson, G. T. Selvan, I. Enoch and P. M. Selvakumar, Appl. Clay Sci., 2017, 135, 234-243.

26 X. Wang, S. Zhou, W. Xing, B. Yu, X. Feng, L. Song and Y. Hu, J. Mater. Chem. A, 2013, 1, 4383-4390.

27 C. Li, J. Wan, E. N. Kalali, H. Fan and D. Y. Wang, J. Mater. Chem. A, 2015, 3, 3471-3479.

28 C. Nyambo, E. Kandare and C. A. Wilkie, Polym. Degrad. Stab., 2009, 94, 513-520.

29 M. Zhang, P. Ding and B. Qu, Polym. Compos., 2009, 30, 1000-1006.

30 Z.-Q. Shen, L. Chen, L. Lin, C.-L. Deng, J. Zhao and Y.-Z. Wang, Ind. Eng. Chem. Res., 2013, 52, 8454-8463.

31 Q. Yao, J. Wang and A. G. Mack, U.S. Pat., 9012546, 2015.

32 D.-Y. Wang, A. Das, A. Leuteritz, R. N. Mahaling, D. Jehnichen, U. Wagenknecht and G. Heinrich, $R S C A d v$., 2012, 2, 3927.

33 D.-Y. Wang, A. Das, F. R. Costa, A. Leuteritz, Y. Z. Wang, U. Wagenknecht and G. Heinrich, Langmuir, 2010, 26, 14162-14169.

34 M. Zammarano, M. Franceschi, S. Bellayer, J. W. Gilman and S. Meriani, Polymer, 2015, 46, 9314-9328.

35 W. Xu, A. Wirasaputra, S. Liu, Y. Yuan and J. Zhao, Polym. Degrad. Stab., 2015, 122, 44-51.

36 X. Qian, L. Song, B. Yu, B. Wang, B. Yuan, Y. Shi, Y. Hu and R. K. K. Yuen, J. Mater. Chem. A, 2013, 1, 6822-6830.

37 X. Wang, Y. Hu, L. Song, W. Xing and H. Lu, J. Anal. Appl. Pyrolysis, 2011, 92, 164-170.

38 U. Braun, A. I. Balabanovich, B. Schartel, U. Knoll, J. Artner, M. Ciesielski, M. Döring, R. Perez, J. K. W. Sandler, V. Altstädt, T. Hoffmann and D. Pospiech, Polymer, 2006, 47, 8495-8508.

39 W. Zhang, X. Li, L. Li and R. Yang, Polym. Degrad. Stab., 2012, 97, 1041-1048.

40 Z. Li and R. Yang, Polym. Degrad. Stab., 2014, 109, 233-239. 41 S. Yang, J. Wang, S. Huo, M. Wang, J. Wang and B. Zhang, Polym. Degrad. Stab., 2016, 128, 89-98.

42 W. Zhao, J. Liu, H. Peng, J. Liao and X. Wang, Polym. Degrad. Stab., 2015, 118, 120-129.

43 Q. He, L. Song, Y. Hu, H. Chen and L. Liu, Polym. Degrad. Stab., 2008, 93, 627-639.

$44 \mathrm{~K} . \mathrm{Wu}, \mathrm{Y} . \mathrm{Hu}$, L. Song, H. Lu and Z. Wang, Ind. Eng. Chem. Res., 2009, 48, 3150-3157.

45 B. Perret, B. Schartel, K. Stöß, M. Ciesielski, J. Diederichs, M. Döring, J. Krämer and V. Altstädt, Eur. Polym. J., 2011, 47, 1081-1089.

46 J. W. Gilman, C. L. Jackson, A. B. Morgan and R. H. Jr, Chem. Mater., 2000, 12, 1866-1873. 